\title{
CURVAS DE SECAGEM E AVALIAÇÃO DA ATIVIDADE DE ÁGUA DA BANANA PASSA
}

\author{
MILTON CANO-CHAUCA * \\ AFONSO M. RAMOS ** \\ PAULO C. STRINGHETA ** \\ JOSÉ ANTONIO MARQUES *** \\ POLLYANNA IBRAHIM SILVA ***
}

\begin{abstract}
Curvas de secagem de banana passa foram determinadas, utilizando-se três temperaturas do ar de secagem. Os resultados indicaram que para reduzir o teor de umidade do produto até $23,5 \%$ foram necessários tempos de secagem de 51,36 e 30 horas paras as temperaturas de 50,60 e $70^{\circ} \mathrm{C}$, respectivamente. $\mathrm{O}$ modelo exponencial $\mathbf{U} / \mathbf{U}_{\circ}=$ $\operatorname{exp(-kt)~foi~ajustado~para~os~dados~experimentais~}$ mediante análise de regressão não-linear, encontrandose alto coeficiente de regressão linear. Determinou-se a atividade de água do produto ao longo do processo de secagem para as três temperaturas testadas. Estudou-se a correlação entre a atividade de água e o teor de umidade do produto, determinando-se as isotermas de dessorção da banana passa a $25^{\circ} \mathrm{C}$. Observou-se que a atividade de água diminuiu em função do tempo de secagem e do teor de umidade para as três temperaturas de secagem. Os dados experimentais foram ajustados mediante regressão não-linear ao modelo polinomial e a seguinte equação foi obtida: $\mathrm{U}=-1844,93+7293,53 \mathrm{~A} a-9515,52 \mathrm{~A}^{2}+4157,196 \mathrm{~A}^{3}$. O ajuste mostrou-se satisfatório $\left(R^{2}>0,90\right)$.
\end{abstract}

PALAVRAS CHAVE: BANANA PASSA - SECAGEM; BANANA - ATIVIDADE DE ÁGUA.

* Eng. Alimentos, Dr. Pesquisador, Núcleo de Tecnologia em Armazenagem, Faculdade de Agronomia e Medicina Veterinária, Universidade Federal de Mato Grosso (UFMT), Cuiabá, MT; Bolsista CNPq, FAPEMAT (e-mail: Miltonc9@ hotmail. com).

** Eng. Alimentos, Doutor, Prof. Titular, Departamento de Tecnologia de Alimentos, Universidade Federal de Viçosa (UFV), Viçosa, MG (e-mail: stringap@ufv.br).

*** Estudante de Graduação, Engenharia de Alimentos, Departamento de Tecnologia de Alimentos, UFV, MG. 


\section{INTRODUÇÃO}

Uma das técnicas de preservação de alimento mais antigas utilizadas pelo homem consiste na remoção de umidade dos alimentos pelo processo de secagem. Em relação aos outros métodos de conservação para períodos longos como a refrigeração, a apertização ou tratamentos químicos, a desidratação oferece custo mais baixo e operações mais simples. A remoção de umidade provoca a diminuição da atividade de água do produto, inibindo o desenvolvimento de microrganismos e retardando deteriorações de origem físico-química.

As frutas e vegetais caracterizam-se pelo alto teor inicial de umidade, sendo considerados produtos altamente perecíveis. Portanto, o processo de secagem implica em considerável redução de custo em transporte e manipulação do produto, além de proporcionar efetivo prolongamento de sua vida útil.

O Brasil ocupa o segundo lugar na produção mundial de banana, com área colhida superior a 511 mil ha. Em 2002, a produção de banana foi de 6.357,940 toneladas e tem-se mantido estável nos últimos anos (AGRIANUAL, 2003). No entanto, as perdas desse produto são estimadas em $40 \%$ do volume total da produção (AGRIANUAL, 1999). Apesar de ser colhida em quantidades e qualidade praticamente iguais ao longo do ano, a fruta deve ser consumida em até 25 dias após a colheita.

Justifica-se a aplicação de métodos de conservação do excedente da produção para frutos extremamente perecíveis. A industrialização é, sem dúvida, uma grande alternativa para o aproveitamento integral da banana (CANO-CHAUCA, RAMOS E STRINGHETA, 2002b). Dentre os processos de aproveitamento industrial, a produção de banana passa requer baixo investimento inicial e perspectivas de lucratividade compatíveis com o investimento. O mercado interno está em expansão e o mercado externo continua inexplorado pelo Brasil.

Obtém-se a banana passa mediante secagem natural ou artificial da fruta madura, comumente a partir da banana-nanica e da bananananicão. Elevado teor de açúcares e umidade entre 20 a 25\% caracterizam a banana passa. Facilmente assimilável, pode ser classificada entre os produtos com alto valor alimentício e como boa 
fonte de energia ativa (BANANA..., 1995; CANO-CHAUCA et al., 2002a). Entretanto, a secagem de frutas ainda constitui operação complexa e pouco compreendida, principalmente no que se refere à seleção e controle das condições do processo para a manutenção da qualidade final do produto. De acordo com NIHJHUIS et al. (1996), a preservação da qualidade de frutas desidratadas depende de fatores como características da matéria-prima, temperatura, umidade relativa do ar de secagem e tipo de secador, entre outros. $\mathrm{O}$ emprego de parâmetros inadequados para a desidratação de determinado produto pode causar diminuição de aroma, alterações na cor, textura e sabor, além de perdas de nutrientes.

A exigência dos consumidores tem aumentado e produtos que preservam ao máximo suas características originais estão sendo preferidos. Em nível industrial, isto significa o desenvolvimento de operações que minimizem os efeitos adversos do processamento. Vários estudos sobre curvas de secagem e atividade de água têm sido realizados devido à sua influência na qualidade do produto desidratado. O conteúdo de umidade de determinado sólido pode ser expresso em termos de massa total, base úmida ou massa seca. Ao entrar em contato com o ar quente ocorre transferência de calor do ar para o produto, devido ao gradiente de temperatura existente entre ambos. Simultaneamente, a diferença da pressão parcial do vapor de água existente entre $o$ ar de secagem e a superfície do produto determina a transferência de massa do produto para o ar em forma de vapor de água (NOGUEIRA, 1991).

Apesar da banana passa estar disponível no mercado, a literatura não fornece informações técnicas sobre sua cinética de secagem, atividade de água e as isotermas de dessorção. Estudos sobre esses parâmetros são necessários no intuito de otimizar o processo de secagem e a obtenção de produto com boa qualidade que satisfaça as exigências dos consumidores.

O presente trabalho visou a obtenção de curvas de secagem da banana passa, a determinação da atividade d'água do produto ao longo do processo de secagem, assim como a determinação da isoterma de dessorção. 


\section{MATERIAL E MÉTODOS}

\subsection{MATÉRIA-PRIMA}

Utilizou-se banana Nanica (Musa spp nanica $(A A A)$ ) adquirida no mercado de Viçosa, procedente da CEASA de Belo Horizonte, MG. Para obter produto com qualidade foram escolhidos frutos desenvolvidos, com 34 a 36 mm de diâmetro, de boa maturação e tamanho uniforme (CANO-CHAUCA et al., 2002a).

\subsection{PREPARO DA AMOSTRA PARA A SECAGEM}

Os frutos passaram por três águas de lavagem, após inverção, sendo a primeira com água corrente de boa qualidade. Em seguida, foram lavados com água contendo $50 \mathrm{ppm}$ de cloro residual total pelo período de trinta minutos. Usou-se água corrente para a terceira lavagem. Foram selecionados frutos completamente maduros, com ponto de maturação firme e uniformidade de coloração e sabor. Os frutos foram descascados manualmente, lavados e colocados em solução de ácido cítrico e ácido ascórbico. Concentrações de $0,1 \%$ foram utilizadas para o primeiro tratamento e de 0,3\% para o segundo, durante 20 minutos. Após escorrer a água, as bananas inteiras foram colocadas nas bandejas do secador para serem desidratadas.

\subsection{SECAGEM}

A banana foi desidratada em secador composto pela câmara de secagem com 10 bandejas de $1 \mathrm{~m}^{2}$ cada, câmara "plenum" com conjunto de queimadores e chama de gás GLP, registro de gás para a linha principal e para a linha piloto, inversor de freqüência, ventilador responsável pela impulsão do ar ambiente para o sistema de aquecimento até a câmara de secagem e duto na saída para medir a vazão do ar. Realizou-se a secagem nas temperaturas de 50, 60 e $70^{\circ} \mathrm{C}$ e velocidade fixa do $\operatorname{ar}(1,5 \mathrm{~m} / \mathrm{s})$ sobre as frutas, sendo as bananas colocadas lado a lado nas bandejas. Para cada tratamento foram realizadas três observações, totalizando nove testes experimentais. Determinou-se a vazão total do ar no duto na saída do secador por meio de anemômetro de fio quente. As medições de temperatura do 
ar de secagem ocorreram por meio de termômetro fixado na entrada da câmara de secagem. Para o acompanhamento da perda da umidade, durante os testes de secagem, foram realizadas pesagens do produto no início do teste e, posteriormente, em intervalos de 3 horas até que fosse atingido o teor de umidade desejado (entre 20 e $25 \%$ ). Ao final da secagem, amostras de banana foram retiradas para determinação do teor umidade final.

\subsection{DETERMINAÇÃO DA ATIVIDADE DE ÁGUA}

Mediu-se a atividade de água dos frutos durante o processo de secagem utilizando aparelho AQUALAB CX2T de leitura direta. Para tais medições foram coletadas amostras em duplicata, a cada 3 horas, ao longo do processo de secagem. As leituras ocorreram a $25^{\circ} \mathrm{C}$, sendo as amostras resfriadas antes da determinação.

\section{RESULTADOS E DISCUSSÃO}

\subsection{CURVAS DE SECAGEM}

As curvas de secagem permitem predizer o momento do processo em que se atinge o teor de umidade desejado e desta forma obter produto de boa qualidade. A Figura 1 mostra a variação no teor de umidade adimensional $\left(U / \bigcup_{0}\right)$ da banana em função do tempo de secagem para as temperaturas de ar de 50, 60 e $70^{\circ} \mathrm{C}$.

O modelo exponencial abaixo (equação 1) foi ajustado para os dados experimentais mediante análise de regressão não-linear.

$$
\frac{U}{U o}=\exp (-k t)
$$

em que:

$\mathrm{U}=$ teor de umidade no tempo $\mathrm{t}$ (\% base úmida).

$U_{0}=$ teor de umidade inicial (\% base úmida).

$\mathrm{k}=$ constante de secagem.

$\mathrm{t}=$ tempo de secagem $(\mathrm{h})$. 
FIGURA 1 - CURVAS DE SECAGEM EXPERIMENTAIS E CALCULADAS PELO MODELO EXPONENCIAL PARA AS TEMPERATURAS DE 50, $60 \mathrm{E} \mathrm{70}{ }^{\circ} \mathrm{C}$

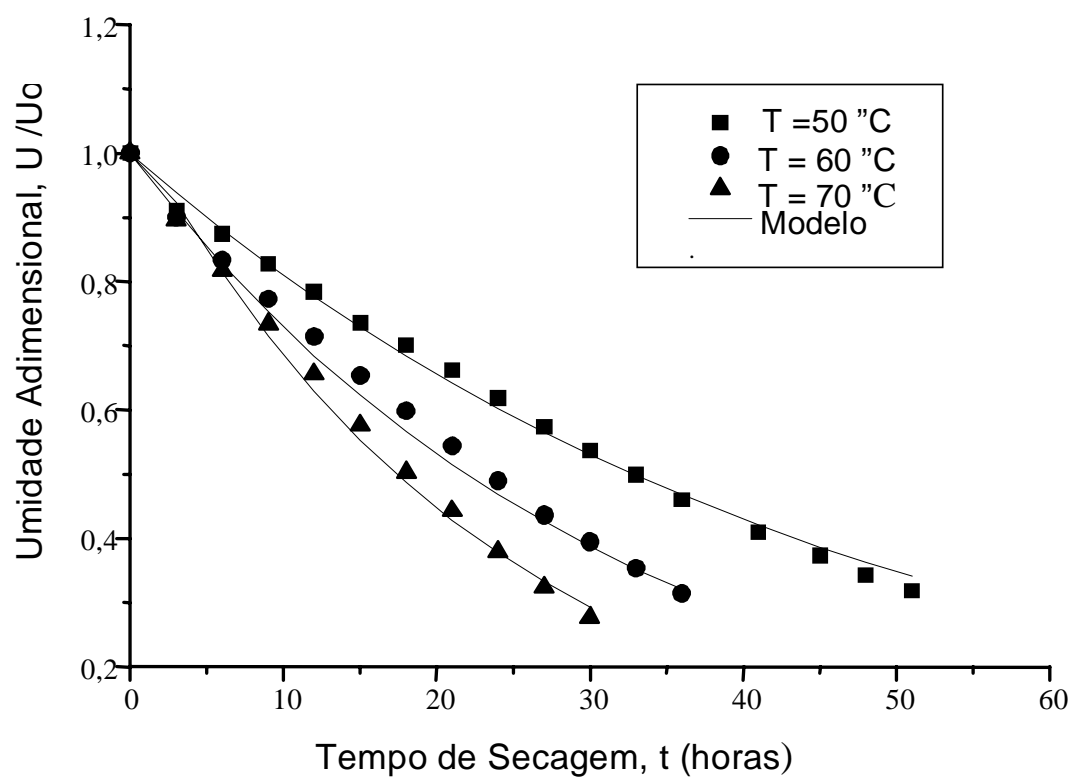

O modelo exponencial ajustado aos dados experimentais, significativo ao nível de probabilidade de $99 \%$, resultaram nas equações 2 , 3 e 4 :

Para a secagem a $50^{\circ} \mathrm{C}$

$$
\frac{U}{U o}=e^{-0,040 t} \quad \mathrm{R}^{2}=0,995
$$

Para a secagem a $60^{\circ} \mathrm{C}$

$$
\frac{U}{U o}=e^{-0,0316 t} \quad \mathrm{R}^{2}=0,995
$$


Para a secagem a $70^{\circ} \mathrm{C}$

$$
\frac{U}{U o}=e^{-0,0211 t} \quad \mathrm{R}^{2}=0,993
$$

Para atingir teor de umidade adimensional de 0,3 (correspondente a $23,5 \%$ em base úmida) são necessários tempos de 51,36 e 30 horas de secagem para as temperaturas de 50,60 , e $70^{\circ} \mathrm{C}$, respectivamente (Figura 1).

Para o tempo de 30 horas de secagem, os valores do teor de umidade para cada temperatura são diferentes e apresentam o mesmo comportamento ao longo do todo o processo de secagem. Isso se deve ao fato de que quanto maior a temperatura do ar maior a taxa de secagem (TRAVAGLINI et al., 1993). A diferença no teor de umidade no início não é muito elevada, porém aumenta conforme o tempo de secagem. No início do processo as diferenças são menores, pois parte significativa da umidade está livre na superfície da banana e nesta forma é facilmente removida. Para os demais tempos de secagem, as diferenças aumentam em virtude da resistência interna ao transporte de umidade. Nesse período, a água interage com os grupos polares das moléculas dos constituintes. Assim, quanto maior a temperatura mais facilmente a água é removida.

De acordo com a literatura consultada, a banana seca ou banana passa deve apresentar teor de umidade entre 20-25\% (BANANA, 1995; PRODUÇÃO..., 1995; TRAVAGLINI et al., 1993). Tendo as curvas de secagem pode-se determinar o tempo de secagem adequado para esse teor de umidade.

\subsection{ATIVIDADE DE ÁGUA}

$\mathrm{Na}$ Figura 2 consta a variação da atividade de água ao longo do período de secagem para as três temperaturas $\left(50,60,70^{\circ} \mathrm{C}\right)$. Na temperatura de $50^{\circ} \mathrm{C}$, a atividade de água atinge o valor 0,76 depois de 51 horas de secagem. Por outro lado, para as temperaturas de 60 e $70^{\circ} \mathrm{C}$ foram atingidos valores de 0,70 e 0,69 após 36 e 30 horas de secagem, respectivamente. 


\section{FIGURA 2 - CURVAS DE ATIVIDADE DE ÁGUA DA BANANA EM FUNÇÃO DO TEMPO DE SECAGEM PARA AS TEMPERATURAS DE 50, $60 \mathrm{E} \mathrm{70}$ С}

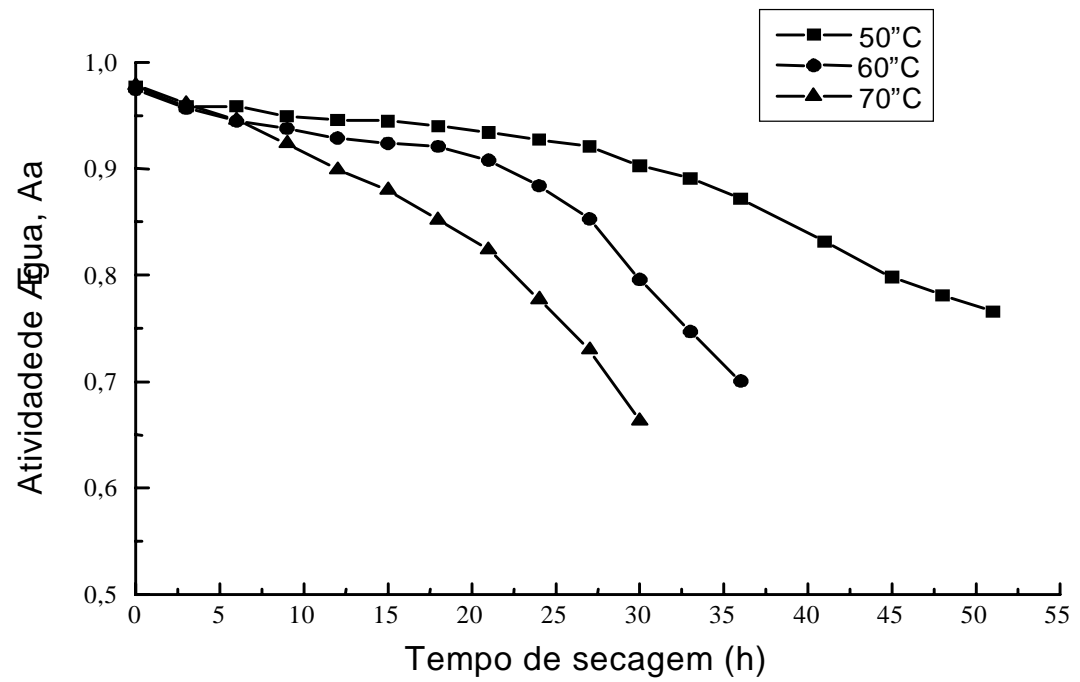

Observou-se que para o mesmo tempo de secagem, a atividade de água do produto aumenta com a diminuição da temperatura. Por outro lado, para temperatura fixa a atividade de água diminui com o tempo de secagem. Tal fato está relacionado com a perda de água durante 0 processo de secagem. Para temperaturas mais elevadas a taxa de evaporação de água é maior, influenciando o teor de umidade e conseqüentemente a atividade de água do produto (SILVA, 1995; TRAVAGLINI et al., 1993).

Os dados da atividade de água obtidos para a banana passa enquadraram-se na faixa de produtos com umidade intermediária. Segundo ERICKSON (1982), esses valores geralmente estão entre $0,60-0,84$ a $25^{\circ} \mathrm{C}$. Tratando-se de produto com umidade intermediária a banana passa é pouco susceptível a deterioração de origem físicoquímica e microbiológica. 


\subsubsection{Relação entre a atividade de água e o teor de umidade}

A atividade de água sofre alterações conforme o teor de umidade do produto como pode ser observado na Figura 3. Para os três níveis de temperatura de secagem $\left(50,60\right.$ e $\left.70^{\circ} \mathrm{C}\right)$ a atividade de água aumentou em função do teor de umidade do produto. Resultados similares foram obtidos por McLAUGHLIN e MAGGE (1998), estudando as isotermas da batata para as temperaturas de 30,45 e $60^{\circ} \mathrm{C}$.

FIGURA 3 - ATIVIDADE DE ÁGUA EM FUNÇÃO DO TEOR DE UMIDADE DA BANANA PARA AS TEMPERATURAS DE SECAGEM DE 50, 60 E 70ㅇ

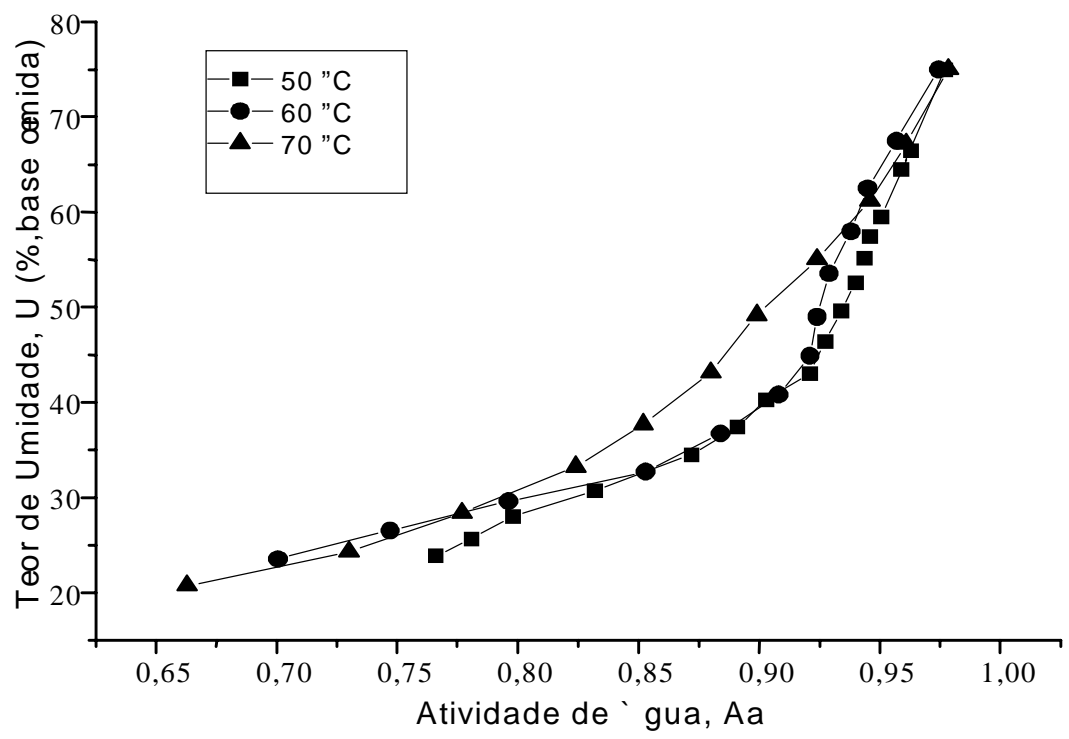

A Figura 4 mostra a isoterma de dessorção da água da banana, obtida a $25^{\circ} \mathrm{C}$ para o produto desidratado em temperaturas de secagem de 50,60 e $70^{\circ} \mathrm{C}$. O modelo polinomial (equação 5) foi ajustado aos dados experimentais pela análise de regressão não-linear, significativa ao nível de $99 \%$ de probabilidade.

$$
U=-1844,93+7293,53 A_{a}-9515,52 A_{a}{ }^{2}+4157,196 A_{a}{ }^{3} \quad R^{2}=0,960
$$


Verificou-se que o modelo polinomial de terceiro grau ajustou-se bem aos dados experimentais conforme a equação 5, plotada na Figura 4. O comportamento da isoterma de sorção é sigmoidal, assim como para a maioria dos alimentos. No caso da banana está apresentada para a faixa de atividade de água de 0,66 a 0,98, sendo a água livre removida durante o processo de secagem.

\section{FIGURA 4 - ATIVIDADE DE ÁGUA EXPERIMENTAL E CALCULADA PELO MODELO POLINOMIAL A 25ㄷ, EM FUNÇÃO DO TEOR DE UMIDADE}

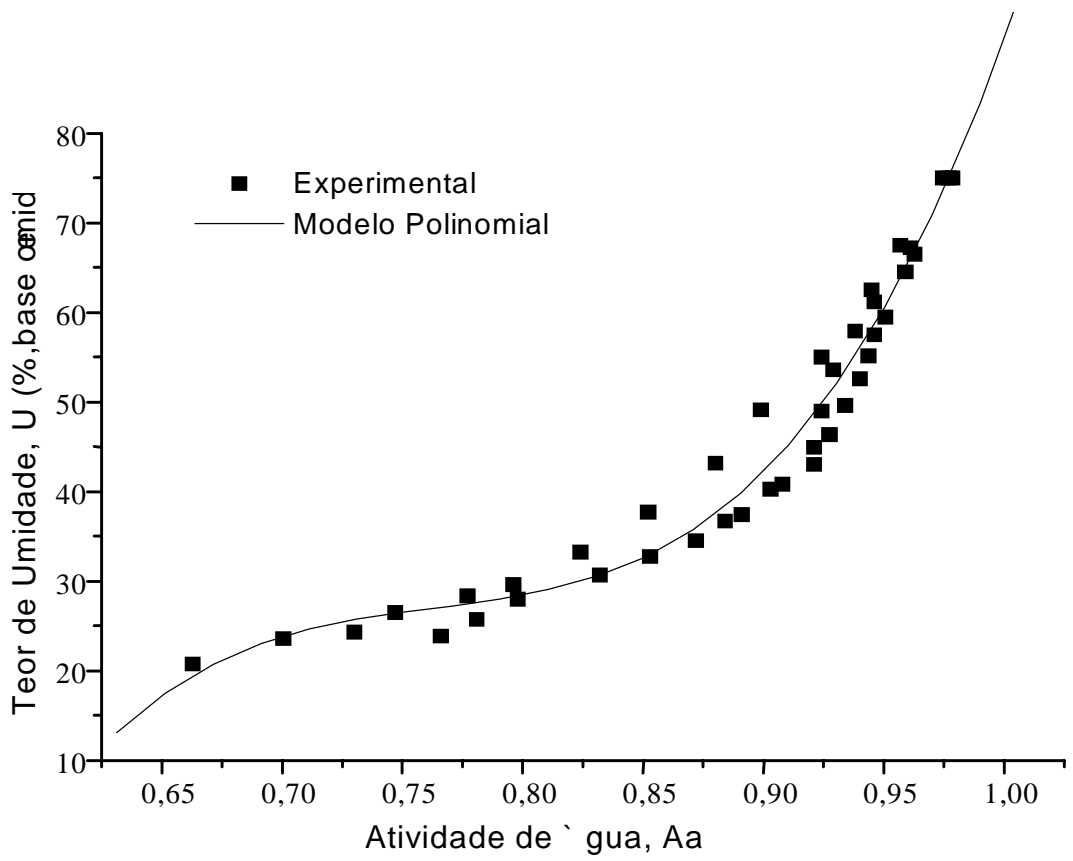

\section{CONCLUSÃO}

Os tempos de secagem da banana para atingir teor de umidade entre 23 a $25 \%$, utilizando as temperaturas de ar de 50, 60 e $70^{\circ} \mathrm{C}$, foram de 51,36 e 30 horas, respectivamente.

O processo de secagem de banana passa pode ser descrito pelo modelo exponencial da forma: 


$$
\frac{U}{U o}=\exp (-k t) \text { no qual: } k=0,04\left(50^{\circ} \mathrm{C}\right), k=0,0316\left(60^{\circ} \mathrm{C}\right), k=0,0211\left(70^{\circ} \mathrm{C}\right)
$$

A atividade de água da banana variou em função do tempo de secagem, tendo aumentado com a diminuição da temperatura durante o mesmo período de tempo. Para temperatura fixa a atividade de água diminuiu com o aumento do tempo de secagem.

O teor de umidade da banana variou com a atividade de água segundo o modelo polinomial:

$$
U=-1844,93+7293,53 A_{a}-9515,52 A_{a}^{2}+4157,196 A_{a}^{3} \quad\left(R^{2}=0,960\right) .
$$

\begin{abstract}
DRYING CURVES AND WATER ACTIVITY EVALUATION OF THE BANANAPASSES

Banana drying curves were determined by utilizing three drying air temperatures. The results indicated that to reduce the moisture content of the product until $23.5 \%$ it were necessary drying times of 51,36 and 30 hours for temperatures of 50,60 and $70^{\circ} \mathrm{C}$, respectively. The exponential model $\mathrm{U} / \mathrm{U}_{0}=\exp (-\mathrm{kt})$ was adjusted for the experimental data by means of non linear regression analysis, and a high coefficient of linear regression was found. The water activity of the product was determined throughout the drying process for the three tested temperatures. The correlation between the water activity and moisture content of the product was studied, and the sorption isotherms were determined at $25^{\circ} \mathrm{C}$. It was observed that the water activity decreased in function to the drying time and moisture content for the three drying temperatures. The experimental data were adjusted by means of non linear regression to the polynomial model and the following equation was obtained: $U=$ $1844.93+7293.53^{A}{ }_{a}-9515.52 A_{a}{ }^{2}+4157.196{ }_{a}^{A}$. The final adjust was satisfactory $\left(R^{2}>0.90\right)$.
\end{abstract}

KEY-WORDS: BANANA-PASSES - DRYING; BANANA - WATER ACTIVITY.

\title{
REFERÊNCIAS
}

1 AGRIANUAL. Anuário da agricultura brasileira. São Paulo: FNP Consultoria \& Comercio, 1999. 512 p.

2 AGRIANUAL. Anuário da agricultura brasileira. São Paulo: FNP Consultoria \& Comercio, 2003. 521 p. 

Campinas, SP: Fundação Cargill, 1998. 236 p.

BANANA: cultura, matéria-prima, processamento e aspectos econômicos. Campinas: ITAL, 1995. 302 p. (Série Frutas Tropicais, 3).

5 CANO-CHAUCA, M.; RAMOS, A.M.; STRINGHETA, P.C.; MINIM, V.P.; ANDRADE, N.J. Análisis físico-química, sensorial y microbiológico de la banana pasa. Alimentaría, v. 329, p.119-122, 2002a.

6 CANO-CHAUCA, M.; RAMOS, A.M.; STRINGHETA, P.C. Color and texture evaluation during banana drying (Musa spp nanica $(A A A)$ ). Alimentaria, Madrid-España, v.339, p.153-158, 2002b.

7 ERICKSON, L.E. Recent developments in intermediate moisture foods. Journal of Food Protection, Ames, v. 45, n. 5, p.484-491, 1982.

8 McLAUGHLIN, C.P.; MAGEE, T.R. The determination of sorption isotherm and the isosteric heats of sorption for potatoes. Journal of Food Engineering, v. 35, p.267-280, 1998.

9 NIHJHUIS, H.; TOORRIG, E.; LUYTEN, H.; RENE, F.; JONES, P.; FUNEBO, T.; OHLSSON, T. Research needs and opportunities in the dry conservation of fruit and vegetables. Drying Technology, v.14, n.6, p.1429-1457, 1996.

NOGUEIRA, R.I. Estudos dos fenômenos de secagem da banana: parâmetros ótimos na obtenção de banana passa. Campinas, 1991. 158 p. Dissertação (Mestrado em Engenharia Agrícola), Universidade Estadual de Campinas.

PRODUÇÃO de banana passa. Brasília: Ministério da Agricultura, da Abastecimento e da Reforma Agrária, Secretaria do Desenvolvimento Rural, 1995. 30 p. (Série Perfis Agro-industriais, 5).

12 SILVA, JUAREZ. S. Pré-processamento de produtos agrícolas. Juiz de Fora: Instituto Maria, 1995. 250 p.

13 TRAVAGLINI, D.A.; NETO, M.P.; BLEINROTH, E.W.; LEITÃO, M.F. Bananapassa: princípios de secagem, conservação e produção industrial. Campinas: ITAL/Rede de Núcleos de Informação Tecnológica, 1993. 73 p. (Manual Técnico 12). 